\title{
A Novel super voxel-based 3D segmentation method for Irritable Bowel Syndrome
}

\author{
Adithya Pothan Raj V, Dr. Mohan Kumar P
}

\begin{abstract}
The main aim of this research paper is to implement a model-driven machine learning based adaptive $3 D$ Segmentation Scheme for detecting the IBS (Irritable bowel syndrome) disease. This algorithm taking into account by endoscopy driven visual images for the purpose of machine analyzing and convert that $2 D$ RGB coordinates into $3 D$ RGB coordinates for improving the accuracy of the segmentation. In previous segmentation schemes, the IBS images are obtained by the use of ultrasound imaginary technique, but the main issue of the imaginary was the noise present in the images. We are overcoming this issue by applying the endoscopy images. Adaptive smoothing technique used in pre-processing stages with neighboring pixel reference. The feature data extraction stages estimate the shape and color and region-based features for segmentation. The proposed scheme performance with our 50 image Database shows that the results accuracy of proposed system outperforms multiple conventional segmentation methods.
\end{abstract}

Keywords: Image Segmentation, 3D image Segmentation, Irritable Bowel Syndrome, Ant Colony Optimization, Adaptive Thresholding

\section{INTRODUCTION}

Image segmentation is among the important systems of the virtual image processor. Image segmentation gets significant picture main features, consisting of edge, areas, and many others. For more image identification, evaluation, and comprehension. The generally utilized image segmentation method derives from the limit, or region, or edge, and many others. The Watershed transformation predicated on morphological refurbishment is an average technique of segmenting an image by the basis of region distention, and extensively used on many of these fields while intelligent transport strategy, medical image evaluation, remote control prying, floatation procedure, and many others. In latest times, a whole lot of analysts suggested a whole slices of segmenting strategies of Image in styles of uses, like the transformation by morphological technique, transformation by watershed approach etc. Nevertheless, it was expressed that a whole lot of edge detection, area segmentation algorithms, there is absolutely none of the technique of segmenting image is good to all image types. Consequently, the analysis for segmenting image continues to be becoming deepening. Picture segmentation continues to be on the study hotspot. Transforming morphologically uses a couple of impartial

Revised Version Manuscript Received on April 19, 2019.

Adithya Pothan Raj V, Research Scholar, School of Computing, Sathyabama Institute of Science and Technology, Chennai - 600 119, Tamil Nadu, India.apr1991@ rediffmail.com

Dr. Mohan Kumar P, Professor, Department of Information Technology,Jeppiaar Engineering College, Chennai - 600 119, Tamil Nadu, India.mohankumarmohan@gmail.com procedures, principles, and the numerical protocol to spell it out over attribute of image. Transforming morphologically can effectively get oblique dimensions of varied orderly variables, and the important features of the image get characterized through the use of numerical solutions based on collection factors and stochastic procedures. Watershed transformation protocol is usually a numerical morphology segmentation manner designed recently, provided a very wide variety of functions in design and technology, areas. Watershed transform concept can effectively get the image advantage throughout linked closed models, it is therefore usefully an area for image segmentation criteria.

The RYKW model which is known as a continuous release, as if the hyperkeratotic grazes created up over cells of white bloodstream is also additionally protected. The junction between epithelial cells is usually certainly an area wherein inter-cell junctions (limited junctions) are created, to enhance the chemical substances' float. Features possess currently been released within the books to judge digestive tract permeability. Cell Junction Improvement (CJE), a fluorescein construct-up among two epithelial cells symbolizing reduced tight-junction proteins before breakage of the greatest basal junction, and Florida: a fluorescein plume interesting in the lumen addressing inadequate apposition between surrounding cells. A covering of cells features from the esophagus to the rectum, gaming an inbuilt positioning in preventing gain access to environmental adversarial parts that could purpose contaminants. They may in fact outstandingly surpass the antique strategies within the needed person function and the high-quality of the following segmentation. Specifically, for IIP, 3 features possess currently been explained: cell Drop-out (CDO), shedding of an enterocyte into the luminal region.

IBS is caused consequently due to the debt over bloodstream or by the circulation of blood in veins, or due to growth of cancerous cells. It may end up being connected with tumors, attacks etc. Reduced epithelial hurdle feature is definitely present in irritable colon disease (IBD), irritable colon symptoms (IBS), Crohn's disease and IBSative colitis. Contrarily, there cannot become discovered any picture analysis strategies which may be employed with no outside control and guaranteed outcomes. Especially, the digestive tract epithelium could end up being the biggest mucosal ground, controlling the transit of macromolecules. Cell Junction Improvement (CJE), a fluorescein construct-up among two epithelial cells symbolizing damaged 
tight-junction proteins before breakage of the greatest basal junction, and Florida: a fluorescein plume participating in the lumen addressing inadequate apposition between nearby cells. Damaged epithelial buffer feature can be present in irritable colon disease (IBD), irritable colon symptoms (IBS), Crohn's disease and IBSative colitis. Medical experts make use of this sort of type of RYK model (purple-yellow-dark) as a descriptive gadget.

Types of Peptic Irritable colon symptoms There not necessarily unusual types of Peptic Irritable colon symptoms:

Irritable bowel syndrome (IBS) is usually many signs-such as ache or discomfort in your abdomen and modifications in your bowel movement patterns-that occur collectively. Medical examiners in the self-self-discipline of dermatology will the acceptance of the belly abrasion specifically predicated on noticeable notice of the swollen areas and the information of the macroscopic features. Medical specialists make use of this form of kind of RYK copy (purple-yellow-dark) as a descriptive gadget. Around is a wide variety of intermediary level visioning complications such as type shaped from a synopsis, stereo system program and object monitoring might make use of respected segmentation methods of make sure that the area of the hobby is certainly generally disguised from all of those additional pictures. Picture segmentation contains a few considerable applications within the field of medical image resolution. The mucosa of the gastrointestinal tract represents the primary obstacle between the internal body and the surface area globe. IBS, tumors, constant injuries or grazes credited to arterial deficiency which primarily have got a nonhomogeneous structure of yellow colored fibrin, crimson granulation, and also dark necrotic eschar (scratch tissue) cells. If pathological circumstances arrive, the permeability could become sped up and as well small epithelial integrity is normally certainly experienced. Today, centered on the arbitrary walker and chart lower a range of effective interactive picture segmentation strategies possess been totally suggested. Specifically, for IIP, 3 features possess currently been referred to cell Drop-out (CDO), falling off an enterocyte into the luminal region; Segmenting image contains the few intensive solicitations within the field of resolving medical images. This idea in truth suggests us that the most effective test depends after at the medical expert's come across and perceptual value. The junction between epithelial cells is usually certainly a location wherein inter-cell junctions (limited junctions) are produced, to increase the chemical substances' float. It has been requested for noticing specific photo editing. The long term release could end up being identified as RYKW model since the hyperkeratotic gashes which have been created over white bloodstream cells is additionally safeguarded. This hurdle is usually generally created through a dual level of lipid cells, showing a strong level of level of resistance to consuming drinking water-soluble parts. It could become linked with tumors, consequently forth and attacks. Several computational eye-sight algorithms may as well be benefitted on or after the living of accurate and in effect picture strategies of segmentation. The mucosa of the gastrointestinal tract represents the primary barriers between the internal body and the surface area globe. Some of the totally computerized

segmentation strategies are present and are also becoming constantly ameliorated. There is an array of intermediate level vision difficulties such as type shaped from a synopsis, stereo system program and object monitoring might make use of respected segmentation methods of make certain that the area of the hobby is definitely generally disguised from all of those various other pictures. Features possess currently been released within the materials to judge digestive tract permeability. A covering of cells functions from the esophagus to the rectum, playing an inbuilt positioning in halting gain access to environmental adversarial elements that could objective contaminants. This junction's permeability can be effective, in stage with diet condition, humoral or sensory alerts, and inflammatory mediators, among others.

IBS is caused consequently of the debt in bloodstream blood flow in veins, or as a result of a cancerous growth. If pathological circumstances arrive, the permeability could end up being expanded and as well small epithelial integrity is certainly experienced. Especially, the digestive tract epithelium could become the biggest mucosal surface area, controlling the transit of macromolecules. As a result, semiautomatic photo segmentation strategies are getting status a few of the users which enables in resolving moderate and hard segmentation careers by the strategy to little work. Medical examiners in the self-self-discipline of dermatology will the popularity of the tummy abrasion specifically predicated on obvious discover of the swollen areas, and the analysis of the macroscopic features. Its miles requested the particular notice of technological photographs and for editing the photos. Currently, structured on the arbitrary walker and chart lower a range of effective interactive picture segmentation strategies possess been suggested. Many algorithms of computational eye-sight benefits since the existence of effective and accurate picture strategies pf segmentation. This idea, in reality, suggests us that the most effective information depends on when at the medical expert's face and perceptual enjoy. Irritable bowel syndrome (IBS) is definitely many signs-such as ache or discomfort in your abdomen and modifications in your bowel movement patterns-that occur collectively. This hurdle is definitely normally produced through a dual level of lipid cells, delivering a powerful level of level of resistance to consuming drinking water-soluble elements. IBS, tumors, regular pains or gashes credited to arterial inadequacy generally got a nonhomogeneous structure of yellow colored fibrin, granulation in violet, and dark necrotic eschar (scratch tissue) cells. This junction's permeability is normally effective, in stage with the eating condition, humoral or sensory alerts, and inflammatory mediators, among others.

Types of Peptic Irritable colon symptoms there usually are unusual types of Peptic Irritable colon symptoms: They might exceptionally exceed of age strategies within the obligatory person function and also top eminence of ensuing segmentation. Some of the totally computerized segmentation strategies are available and are also getting continually ameliorated. Consequently, semiautomatic photo 
segmentation strategies are obtaining status a few of the users which enables in resolving moderate and hard segmentation responsibilities by the strategy to little work. Contrarily, there won't end up being discovered any picture test strategies which could be employed without the exterior control and made certain outcomes.

- Gastric Irritable bowel syndrome.

- Duodenal Irritable bowel syndrome.

\section{A. Gastric Irritable bowel syndrome}

Those type of Irritable bowel syndrome bureaucracy in the stomach linings. Those Irritable colon symptoms are caused both, as an end result of the contaminants with Helicobacter pylori (L. Pylori) bacteria Helicobacter pyloriH. Soreness occurs whilst the foods continue to be in the stomach. It can also express at this time after consuming. Those type of Irritable colon symptoms bureaucracy in the belly linings. Those Irritable colon symptoms are activated both, as an end result of the contaminants with Helicobacter pylori (L. Pylori) bacteria Helicobacter pyloriH. Discomfort occurs whilst the foods continue to be in the tummy. It can also express at the moment after consuming.

\section{B. Duodenal Irritable bowel syndrome}

These type of Irritable bowel syndrome bureaucracy in the top little gut. Those are also credited to the similar components as within the Gastric Irritable colon symptoms. In case of duodenal Irritable colon symptoms, discomfort happens in the abdomen during the same period as the belly is usually vacant, and the condition enhances after eating.

\section{LITERATURE SURVEY}

Based on the things info is thought to become distributed all over pictures, the complexity in the image segmentation issue may differ significantly. Although some premature performs only consider the picture segmentation of a few pictures owned by the exact strict instance of object, afterward types of segment of image a couple with a lot for a huge selection of the images extracted since distinct target situations. Sadr-Kazemi et al utilize the same section memory foam imageries, directed away which a major algorithm is usually to tag the froth seeds region beforehand. Jianbin Shao highlights the need for froth observing at the most effective limit, which in turn, in case of so much, should certainly grounds under segmentation, then whenever very small, will bring about over segmentation. Wangsheng Yu et al provides a notion of the morphological reshaping the indicators in so that they can enhance the precision of checking. Yuqin Liu et al implements transformation by high-low-hat for pre-processing the obtained image for improve the comparison percentage, developing hence improvised the segmenting precision. Hemin Hao et al suggests an inception based self-modifying technique of segmenting images. This usually could not encounter with a completely convincing end solution, however Yao et al. suggested haphazard centered by forest procedure for acceptable grained distinction. In contrast to standard haphazard forests, designed to use the exact characteristic vector Multimedia Methods Application throughout whole trees concept, the experts allow every node of the tree to study the positioning finally, the supreme feature

discriminating and employ these coupled by means of several learned data since the descendant node to consider. Through the traversal of the tree, data of different regions is usually drawn collectively which in turn outcomes in the ultimate resolution. One limitation of the methodology may be the dependence on a bounding package at the time of the test amount of duration for getting a standardized picture. Yuning Chai suggested a technique called TriCoS. This will solve the co segmentation issue as a result of reducing deficits at the three major distinct sections: the classification level for foreground or the background regularity throughout pictures that belong within similar range, the quantity of picture for spatial continuousness after one by one image, plus the quantity of dataset to splendor among multiple classes. Jun Li recommended the fresh segmentation supervised procedure for hyperspectral remotely noticed picture computer data. This combines unreal and also the spatial data in a structure of Bayesian formatting. Li also utilized the multinomial logistic regression (MLR) protocol for starting with, to understand the ulterior possibility distributions in the unreal data, utilizing a subspace output solution to characterizing sound in a better way and also mixed the pixels extremely. Further, in-text details are included utilizing a multi-level logistical Markov-Gibbs Markov random discipline prior. At last, an optimum segmentation by posteriori is effectively calculated by $\alpha$-Expansion minimum algorithm for cut-structured figure improvement. Chunming Li [10] represents the significant problem for picture segmentation. For resolving the issue of outdated region based and as it characteristically depend upon algorithms for segmenting an image which concentrates in the parts of attention, and frequently be unsuccessful to provide segmentation accuracy effects because of the concentration in homogeneity. Li [10] proposes a novel region based method for segmenting picture, which will be enables to handle strength in homogeneities in the segmenting. The writer on behalf of the style of images with strength in homogeneities, and develop a property of clustering area intensity of the intensity images. This defines an area for clustering requirement function for the image intensities in a community of every point first. After that integration, the neighborhood criterion clustering efficiency with respect to a nearby middle for giving worldwide requirements of segmenting images. The technique anticipated in [10] can concurrently perform image segmentation and calculate the tendency subject, and the predicted preconception field can also be utilized for intensity in the in homogeneity modification.

\section{PROBLEM DEFENITION}

The primary issue of the earlier research methods was not focusing about the 3D segmentation also the image acquisition uses the Ultrasound imaginary system in most time the image produced by the ultrasound imaginary system contains the Noisy Coordinates, which is prospects to the poor performance in the segmentation.

In the standard system for noise elimination, the majority of the system uses the filter centered noise elimination scheme. 
But filtration system structured sound removal plan the disease feature will become reduction credited to the house of filter systems. We are applying the nearest neighbors centered picture quality improvement structure in which the lossy coordinates will obtain improved in conditions with their nearest coordinates feature which will enhance the general quality of the picture.

In most feature extraction systems focusing on the color based feature extraction in our work we are applying the feature extraction based on the color, region, and form of the image, it will enhance the overall accuracy of the program with segmentation.

\section{PROPOSED METHODOLOGY}

The grey amount of processing pixel is changed through average amount of 8-neighbor aspects. There are many high-pass filter systems, such as for example average filtration system and averaging filtration system, could be adopted intended for minimizing the irrelevant pixels in abnormal scanned picture. The median filter might greatly minimize noise, however the bounds info and structure behavior are also distorted, which in turn might help it be hard on removing shape data. Therefore, for reducing irrelevant and conserving the thing details, we apply mean filter to get rid of the stipple noisiness. The amount of pixel in binary picture is just 0 (black color) and 255 (white)

We are able to fix a limit for the picture in gray to separate almost every ideal pixels keen on dark and white colored, and the meaning is thought as like following where the limit; then your image can be an image like binary. By using the limit refinement binaries, the brought image can be less complicated than primary gray image. Then your next process, segmentation, May also uncomplicated to process and identify. For Irritable Bowel Syndrome images, there are several important preferences of the noise filtering algorithms ought to be observed. Initially, usually do not drop the important info many of these as object boundaries and comprehensive components. Further, decrease the irrelevant features efficiently over the homogeneous regions. As the quality higher Irritable Bowel Syndrome pictures are preferable to other Irritable Bowel Syndrome images, like $\mathrm{X}$-rays and ultrasounds, the traditional easy sifting and the ready distribution could be useable to lessen noisiness prior to segmenting. Consequently, the predigesting in the work was used a few strategies: mean filtering and binary threshold leveling.

\section{V.TUMOR SEGMENTING BY ANT COLONY OPTIMIZATION}

The suggested ant colony optimization (ACO-based) trier is utilized for monitoring the IBS shape in the abdominal Computed tomography pictures. The protocol is usually controlled as Ant's analysis through a preliminary startup level and also finishes line level. Where "S" may be the amount of sequences and "A" may be the amount of Ant's that may upgrade the pheromone in every circular formation. The ant colony optimization - centric trier acquired the enhanced shape for "A" ant's that may monitor the portion clefts on the sagittal look at abdominal Computed tomography pictures. At initial, source picture is a sagittal watch of abdomen Computed tomography pictures. Further, the choice is to begin the level, end-stage and initializing step by pheromone table. Further, the procedure identified many applicant shapes that select the finest shape to revise pheromone for every phase. At last, we are able to utilize the upgrading guidelines of pheromone and discover the ideal shape that could end up being the abdomen tumor. The outcomes of abdomen majority segmentation by ACO-based trier.

\section{POSTPROCESSING FOR OUTSIDE CONTOUR EXCISION}

In the earlier process, the areas of segmentation have associated shape by the overall figure (outdoors) and abdominal (indoors). Nevertheless, we tend to adjust the need to get the shape of abdominal through this work, thus we recommended the next technique to decrease the external contour of the body for any segmented pictures.

\section{TUMOUR RECONSTRUCTION}

Generally, there are 30 constant pictures for every function, so we're able to merge the data of $2 \mathrm{D}$ constant pictures keen on a three dimensional feature image. In this work, we have utilized the three dimensional controlled lattice solution to restore the three dimensional quantity. The reliability upon the shape pictures following the ant colony optimization based trier algorithm for segmentation and discovered the resultant associations between your bordering pictures for restoring the round regulated lattices. After that, we utilized a sufficient filtration system for changing the unsoften areas, like for example funneling chops protocol, which is a high-resolution three dimensional area production procedure. For funneling chops procedure, generally, there are twelve surface area filter systems to change the unsmooth areas. The architectural flow of the three dimensional regulated lattice technique is demonstrated. The two illustrations for three dimensional refurbishment (with the tumor) through regulated lattice technique. 


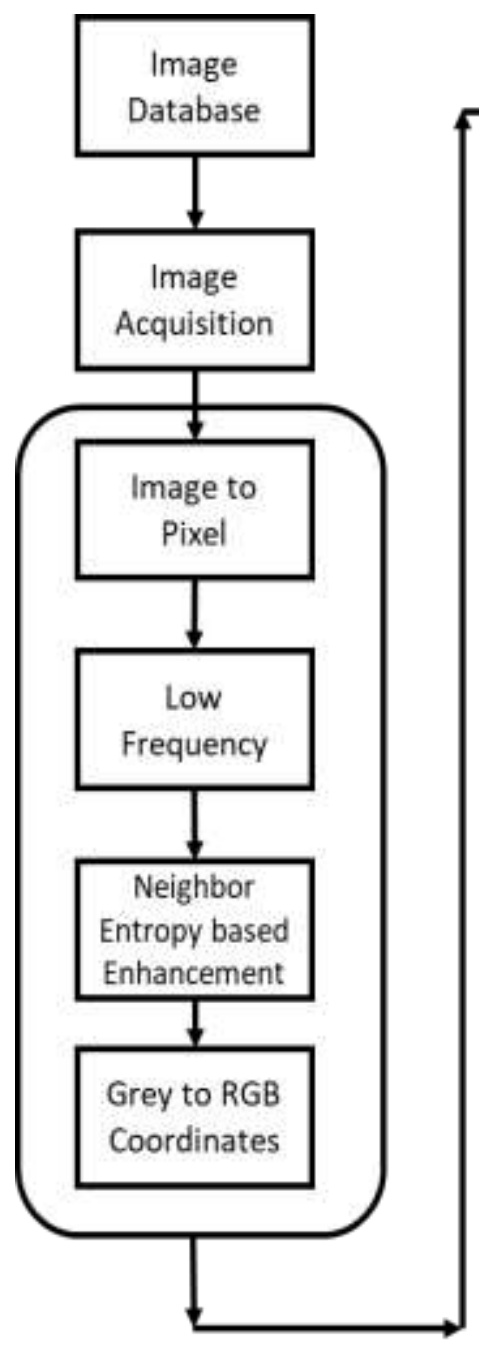

\section{RESULTS}

By the evaluation, generally there was 20 evaluation circumstances and every single circumstance features 515 constant challenging photos with an axial view. Almost all of the images experienced been lately ready by recommended restoration and segmentation system. The consequences obtained by correctness, contact to brain and the "F" measure is generally laid out. The assessments display our approach final results actually even more delighted general overall performance. Since all of the highlights in the abdominal were maintained, we can combine each of the images segmented to restore a three dimensional element for processing an additional declaration and evaluation over the possibilities. The proposed methodology is expressed in Figure. 1 below.

\section{CONCLUSION}

In this research, we will combine the computer-aided diagnosis (CAD) program to offer the doctor the considerably more dependable eyesight for preoperative figuring out and analyzing. We improve, refine a new technique for segmenting abdominal and IRRITABLE BOWEL SYNDROME to assist the health practitioner in figuring out and analyzing preparatory. We as well restore the extracted abdominal and tumor areas to a three-dimensional (3D) picture component to present physicians the even more dependable perspective. The recent outcomes display that the 
6. GuangJian Tian, Yong Xia, Yanning Zhang, and Dagan Feng, "Hybrid Genetic and Variational expectation-Maximization Algorithm for Gaussian-Mixture-Model-Based Brain MR Image Segmentation", IEEE transactions on information technology in biomedicine, VOL. 15, NO. 3, may 2011 373.

7. Amiya Halder, Nilavra Pathak," An Evolutionary Dynamic Clustering Based Colour Image Segmentation", International Journal of Image Processing (IJIP), Volume (4): Issue (6).

8. Keri Woods. "Genetic Algorithms: Colour Image Segmentation Literature Review", July 24, 2007.

9. Li J, Bioucas-Dias JM, Plaza A (2012) Spectral-spatial hyperspectral image segmentation using subspace multinomial logistic regression and markov random fields. IEEE Trans Geosci Remote Sens 50(3):809-823

10.Li C, Huang R, Zhaohua Ding J et al (2011) A level set method for image segmentationin the presence of intensity inhomogeneities with application to MRI. IEEE Trans Image Process 20(7):2007-2016

11.Fahd M. A. Mohsen, Mohiy M. Hadhoud, Khalid Amin," A new Optimization-Based Image Segmentation method By Particle Swarm Optimization", (IJACSA) International Journal of Advanced Computer Science and Applications, Special Issue on Image Processing and Analysis.

12.Alexei N. Skourikhine, Lakshman Prasad, Bernd R. Schlei," Neural Network for Image Segmentation", the Conference Applications and Science of Neural Networks, Fuzzy Systems and Evolutionary Computation, part of 45th SPIE's International Symposium on Optical Science and Technology, San Diego, Calif., July 31-August 4, 2000. Proc. SPIE, Vol. 4120, 28-35, 2000.

13.Fahd Mohsen, Mohiy hadhoud, Kamel Mostafa, Khalid Amin,"A New Image Segmentation method based on new Particle Swarm Optimization", International Arab Journal of Information Technology Vol. 9, No. 5, September 2012.

14.Mostafa Jabarouti, Moghaddam and Hamid Soltanian-Zadeh," Medical Image Segmentation Using Artificial Neural Networks", Image Analysis Lab., Radiology Department, Henry Ford Health System, Detroit, Iran Michigan, USA.

15.Orlando J. Tobias" Image Segmentation by Histogram Thresholding Using Fuzzy Sets,” IEEE, and Rui Seara, Member, IEEE.

16.Mantas Paulinas, Andrius Ušinskas "A Survey Of Genetic Algorithms Applications For Image Enhancement And Segmentation," Issn 1392 - 124x Information Technology And Control, 2007, Vol.36, No.3.

17.Liu Y, Yuan W, Guo J (2011) On-line palmprint recognition based on wavelet decomposition and high-andlow hat transformation. Appl Res Comput 28(6):2355-2357

18.Sadr-Kazemi N, Gilliers JJ (1997) An image processing algorithm for measurement of flotation froth bubble size and shape distributions. Miner Eng 10(10):1075-1083.

19.Shao J, Chen G (2011) Segmentation of bubble image based on watershed algorithm. J Xi'an Univ Technol 27(2):185-189

20.Indah Soesanti, Adhi Susanto, Thomas Sri Widodo, Maesadji Tjokronagoro "Optimized Fuzzy Logic Application For Mri Brain Images Segmentation," Vol 3, No 5, Oct 2011.

21. Kanchan Deshmukh, B. Ganesh Shinde "Adaptive Color Image Segmentation Using Fuzzy Min-Max Clustering," 4 August 2006.

22.K.Selvanayaki, Dr.P.Kalugasalam” Intelligent Brain Tumor Tissue Segmentation From Magnetic Resonance Image (Mri) Using Meta Heuristic Algorithms," Volume 4, No.

\section{2, February 2013}

23. Yao B, Khosla A, Li F-F (2011) Combining randomization and discrimination for fine-grained image categorization. In: CVPR pp. $1577-1584$

24.Yu W, Hou Z, Wang C et al (2011) Watershed algorithm based on modified filter and marker-extraction. Acta Electron Sin 39(4):825-830

25.A. Fabijanska, "Variance filter for edge detection and edge-based image segmentation," in Proc. International Conference on Perspective Technologies and Methods in MEMS Design (MEMSTECH), pp. 151-154, 2011.

26.M. J. Islam, S. Basalamah, M. Ahmadi, and M. A. S. hmed, "Capsule image segmentation in pharmaceutical applications using edge-based techniques," IEEE International Conference on Electro/Information Technology (EIT), pp. 1-5, 2011.

27.M. SHARIF, M. RAZA, and S. MOHSIN, "Face recognition using edge information and DCT," Sindh Univ. Res. Jour. (Sci. Ser.), vol. 43, no. 2, pp.209-214,2011.

28.Advances in Intelligent Information Hiding and Multimedia Signal Processing, Springer Science and Business Media LLC, 2018.

29. Chii-Jen Chen. "Chapter 35 Image Segmentation for Lung Lesions Using Ant Colony Optimization Classifier in Chest CT", Springer Science and Business Media LLC, 2018.

30. Sungkrityayan Khan, Swarupa Paul, Sambu Shiva Rao, Adithya Krishnareddy. "Segmenting skin ulcers based on thresholding and watershed segmentation", 2015 International Conference on Communications and Signal Processing (ICCSP), 2015.

31.Yanpeng Wu, Xiaoqi Peng, Kai Ruan, Zhikun $\mathrm{Hu}$ "Improved image segmentation method based on morphological reconstruction", Multimedia Tools and Applications, 2016.

32. Chii-Jen Chen, You-Wei Wang. "A Preoperative 3D Computer-Aided Diagnosis System for Lung Tumor", 2011 Fifth International Conference on Genetic and Evolutionary Computing, 2011.

33. Adithya Pothan Raj V, Dr Mohan Kumar P "Defective Tissue Segmentation - A Literature Evaluate of past Ten years", International Conference on Recent Advances in Computer Science and Engineering 2019.

34. Adithya Pothan Raj V, Dr Mohan Kumar P "Defective Tissue Classification - A Literature Evaluate of past Ten years", National conference on Innovative Computing Science and Engineering 2019.

\section{AUTHORS PROFILE}

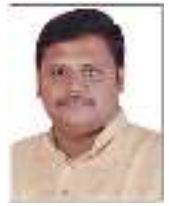

Adithya Pothan Raj. V received the B.Tech. degree in Information Technology from the Anna University, Guindy, Chennai, Tamil Nadu, India in 2012 and the M.E. degree in Computer Science and engineering from Anna University, Guindy, Chennai, Tamil Nadu, India in 2014. He is currently pursuing the Ph.D. degree in School of Computing at Sathyabama Institute of Science and Technology (Deemed to be University), Chennai, Tamil Nadu, India. His area of research interest includes Image Processing, Artificial Neural Networks, Machine Learning and Artificial Intelligence. 
Dr. Mohan Kumar. $\mathbf{P}$ has received his Under-graduation from Manonmaniam Sundaranar University, Tirunelveli, Tamil Nadu, India and Post-graduation from Anna University, Guindy, Chennai, Tamil Nadu, India. He was awarded with Ph.D. degree at Sathyabama Institute of Science and Technology (Deemed to be University), Chennai, Tamil Nadu, India. He is in the teaching field for almost $16+$ years and working as Professor in the Department of Information Technology, Jeppiaar Engineering College, Chennai, Tamilnadu, India. His area of interest includes Image Processing and Image Steganography. 